\title{
Transforming a hospital safety and ergonomics program: a four year journey of change
}

\author{
Vicki J. Missar ${ }^{\mathrm{a}}$, Don Metcalfe ${ }^{\mathrm{b}}$ and Gail Gilmore ${ }^{\mathrm{c}}$ \\ ${ }^{a}$ Aon Global Risk Consulting, 2711 N. Haskell Avenue, Suite 800, Dallas, TX 75204 USA \\ ${ }^{\mathrm{b}}$ Medical Center of Central Georgia, 777 Hemlock St, Hosp Box 127, Macon, GA 31201 USA \\ ${ }^{c}$ Aon Global Risk Consulting1901 Main St. Suite 300, Irvine, CA. 92614
}

\begin{abstract}
The conception of "Patient Safety" being the number one priority at Hospitals can reduce the emphasis on overall employee safety and health. This review examines a hospital's need to improve 24/7 active (i.e., not reactive) coverage, regulatory compliance, as well as the frequency and severity of employee injury losses. It also discusses a journey to integrate and improve safety and ergonomics to achieve these goals. Three approaches used by the ergonomist to create the transformation included: 1) adoption of the safety and ergonomic hazard identification; 2) safe patient handling; and 3) implementation of a $5 \mathrm{~S}$ program. The results of the four (4) year effort at the not for profit, 637 bed, full service, acute-care hospital has shown a steady decline in frequency, reduced waste, and improved housekeeping. Ergonomists can have a key role in transforming Hospital Safety and Ergonomic Programs.
\end{abstract}

Keywords: Hospital, Safety, Ergonomics, 5S, Program, Patient Handling

\section{Introduction}

Transforming a hospital's safety and ergonomics system into a high performing, proactive program can be a daunting task. Given all the moving parts, from outside physicians, on-staff physicians, extensive campuses, satellite locations, emergency care, environmental services, and shortage of nurses, the management of the safety and ergonomic complexities of this industry sector pose unique challenges. In addition, compliance with regulatory elements, patient safety concerns, transition to electronic medical records, and overall quality of care can be taxing to most hospital staff. Thus, the safety and health of employees may take a reduced emphasis.

A private, not-for-profit, 637 bed, full service, acute-care hospital was concerned about improving the overall performance of the safety and ergonomics program to achieve 1) $24 / 7$ coverage, 2) proactive versus reactive status and 3) a "ready state" for any type of compliance and regulator inspections. The primary issues facing this hospital were patient han- dling exposures, a non-urgent safety culture, and minimal systems in place to generate employee participation and involvement in the ergonomics and safety process. This paper will focus on the three (3) primary tools selected by the hospital to improve and reduce its overall exposures. First was the adoption of an ergonomic and safety hazard identification program that transitions the organization from reactionary to a proactive mode. Second, the company focused on a safe patient handling program that would significantly reduce employee lifting exposures associated with patient transfer. And third was the implementation of a $5 \mathrm{~S}$ program that focused on ergonomic exposures as well as general housekeeping practices that were hampering safe working conditions.

\section{Background}

From 2003 to 6/20/2005 the hospital reported over 1,303 work-related incidents for an average of 43 
employee incidents per month. As shown in Table 1, patient handling, furniture/equipment related accidents and slip/falls were the leading loss driver and accounted for $59.85 \%$ of the total claim frequency. Of note were the Furniture/Equipment related claims which comprised a variety of incidents involving employees encountering strains, sprain, lacerations and contusions as a result of equipment. This included injuries from handling equipment such as stretchers and intravenous (IV) poles to handling medication carts, beds and box cutters. In addition this loss category also included falling stock, being struck by or getting fingers caught in closing doors and injuries while reaching for chairs or other equipment. The key departments reporting claims included Surgery, Laboratory, Emergency Center, Nutritional Services, and various Patient Care Floors demonstrating that the issues ran across the hospital and affected employees from nurses to hospital police to maintenance. These claims cost millions in total net incurred costs.

The furniture/equipment injuries in combination with the falls and patient handling, revealed several inherent gaps in the overall ergonomics and safety program. The gaps included a small safety and health staff (3 employees), lack of a system or process to identify ergonomic and safety issues before an event occurred, lack of 24/7 ergonomic and safety coverage across a vast 2.5 million square foot campus, clutter and storage issues creating inherent exposures to not only employees, but patients and visitors as well, and a lack of department directors "walking the talk" to demonstrate their interest and core ergonomic and safety values to employees. The end results was a miss aligned program that needed achievable change to positively reduce the frequency and severity of work-related injuries and improve the overall health and well-being of employees while enhancing the hospital experience for patients and visitors alike.

To affect long term change and move at pace agreeable with the current structure, a Board Certified Professional Ergonomist (CPE) was hired to design, lead, support and/or facilitate the organizational processes and procedures necessary to transform the hospital.
Table 1

Type of claims reported and the corresponding $\%$ to total.

\begin{tabular}{|ll|}
\hline INJURY TYPE & CLOF TOTAL \\
\hline Handling Patient & $24.23 \%$ \\
Furniture/Equipment & $18.76 \%$ \\
Slip/Fall & $16.86 \%$ \\
Other & $8.08 \%$ \\
Lifting & $5.46 \%$ \\
Needlesticks & $5.46 \%$ \\
Foreign Object & $4.28 \%$ \\
Office & $4.28 \%$ \\
BBF Exposure & $2.85 \%$ \\
Push/Pull & $2.38 \%$ \\
Miscellaneous & $7.36 \%$ \\
\hline Grand Total & $100.00 \%$ \\
\hline
\end{tabular}

\section{Program design}

After reviewing the loss trends, interviewing key stakeholders and employees, the ergonomist worked with the safety and health team to devise an overall strategy for success. The strategy design was broken down into three distinct programs to address fundamental ergonomic and safety issues; 1) hazard identification process and protocols (HIPP), 2) patient handling $(\mathrm{PH})$ and 3) 5S, with each program launching in consecutive years. The programs and essential elements designed for each program along with the launch year are listed in Table 2.

\subsection{HIPP}

The first program was the Hazard Identification, Process and Protocols program or HIPP. In reviewing the accident trends and reporting process it was apparent that there was limited efforts around preventing unsafe acts and unsafe conditions. Thus employees lacked a process and the hospital lacked an overall system to help employees report injury-producing exposures that would result in strain, sprain, lifting, slip/fall, contusions, laceration and other injuries. Without early identification and correcting hazards, the hospital would continue on a reactive ergonomics and safety path that would lead to a continuation of accident trends. 
Table 2

Programs implemented, the essential design elements of each program and the year the program was launched.

\begin{tabular}{|c|c|c|}
\hline PROGRAM & ELEMENT & $\begin{array}{l}\text { LAUNCH } \\
\text { YEAR }\end{array}$ \\
\hline HIPP & $\begin{array}{ll}\text { 1. } & \text { Develop a HIPP program } \\
\text { across the hospital enterprise } \\
\text { 2. } \\
\text { Designate HIPP coordinators } \\
\text { to achieve } 24 / 7 \text { coverage } \\
\text { 3. Create a web-based tool for } \\
\text { HIPP tracking } \\
\text { 4. } \begin{array}{l}\text { Report HIPP and other safety } \\
\text { metrics to all employees }\end{array}\end{array}$ & 2007 \\
\hline PH & $\begin{array}{l}\text { 5. Understand the extent of the } \\
\text { program and needs on vari- } \\
\text { ous floors } \\
\text { 6. Implement an effective lift } \\
\text { team } \\
\text { 7. Secure funding for lift } \\
\text { equipment } \\
\text { 8. Implement equipment and } \\
\text { train employees }\end{array}$ & 2008 \\
\hline $5 \mathrm{~S}$ & $\begin{array}{l}\text { 9. Implement a 5S program in } \\
\text { select pilot departments } \\
\text { 10. } \begin{array}{l}\text { Create a train-the-trainer } \\
\text { program for in-house exper- } \\
\text { tise }\end{array} \\
\text { 11. } \begin{array}{l}\text { Roll out success } 5 \mathrm{~S} \text { to other } \\
\text { departments in stages }\end{array}\end{array}$ & 2010 \\
\hline
\end{tabular}

A simple, easy-to-implement system that was user friendly on the front end and credible on the back end (e.g. fixing the reported issues) was essential to the success of HIPP. The vehicle to achieve 24/7 coverage across the enterprise was to train nearly 100 Prevent and Control Officers (PCO) in HIPP. The PCO employees represented a range of job classification including nurses, administrators, lab technicians, supervisors, maintenance, directors, hospital police and others. The PCO employees also covered various shifts and were spread across the hospital's campus. After extensive training in how to identify ergonomic and safety hazards that occurred monthly for 12 consecutive sessions, the employees were then trained on the HIPP reporting system.

The on-line HIPP reporting system, termed "Safety Sleuth" was a web-based, Access program that captured any hazards discovered by the PCO employees. Employees were required to $\log$ into the system and complete the "Safety Sleuth" request form (Figure 1). The employees identified the location of the hazards, completed a facilities request form if necessary and categorized the issue in terms of general safety and ergonomics, environmental, medical equipment, hazardous material, infection control, utilities, and fire safety which match common accident trends. These categories also helped route the issue to the right process owner. For exam- ple, infection control issues were routed to the Infection Control Department, general safety issues were routed to the Safety Department and so on.

To get the program off the ground the employees were trained on the website and given a performance goal of at least finding one ergonomic or safety hazard per month. This performance goal was to help ensure that the PCO employees focused on locating hazards and were held accountable using a measurable metrics. Directors, include the COO, were trained in the program in order to gain their support and help solidify the momentum.

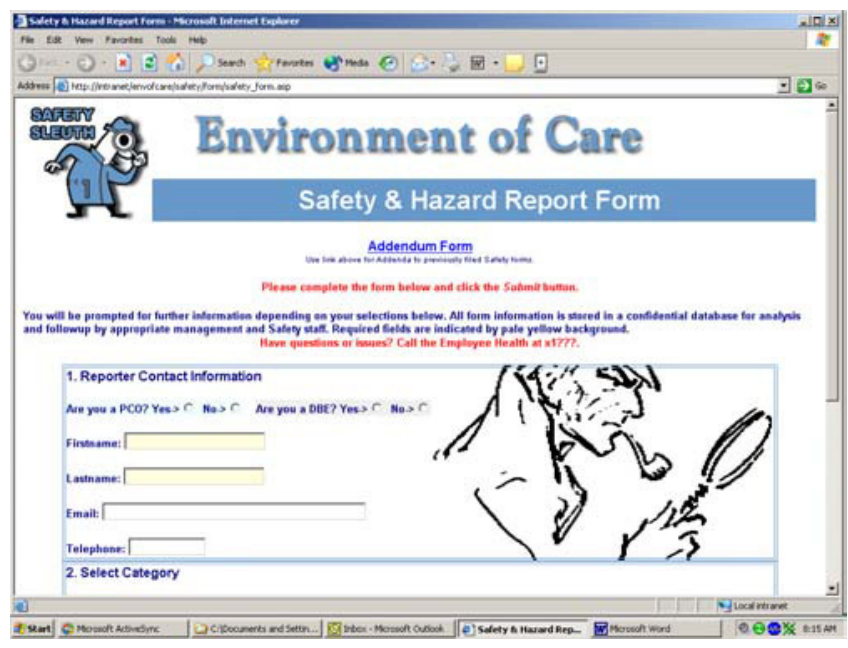

Fig. 1: Section of the HIPP/Safety Sleuth web-based form for tracking issues identified by the PCO employees.

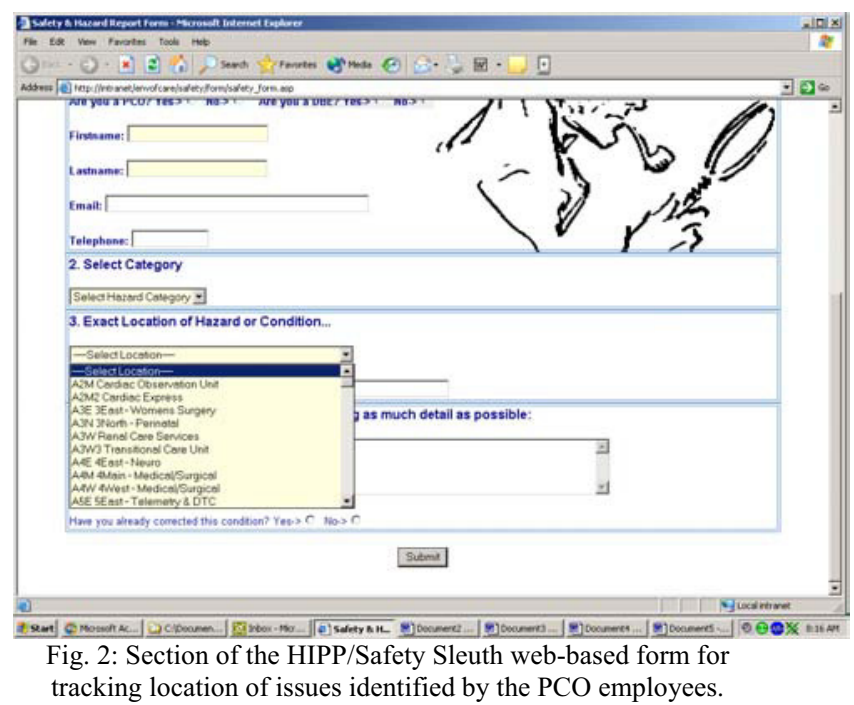

On the back end, the Access program was monitored by the ergonomist and safety team to ensure 
hazards were routed correctly and tracked all the way to closure. The hospital achieved a $92 \%$ closure rate on all hazards identified which is a significant accomplishment. The PCO employees did a solid job identifying hazards each year (Table 3). Note that the number of hazards identified decreased each year which was expected as more exposures were controlled and/or eliminated annually.

Table 3

Number and type of Safety Sleuth on-line entries reported by year

\begin{tabular}{|llll|}
\hline TYPE & 2008 & 2009 & 2010 \\
General Safety and & 821 & 668 & 549 \\
Ergonomics & 379 & 326 & 248 \\
$\begin{array}{l}\text { Slip/Trip/Fall } \\
\begin{array}{l}\text { Total Reports } \\
\text { Submitted }\end{array}\end{array}$ & 1191 & 975 & 888 \\
\hline
\end{tabular}

\subsection{Patient handling}

In January of 2008 the hospital secured a \$1 million grant to implement a safe patient handling program. The ergonomist acted in a consulting role to assist the hospital and the patient handling team in program development and vendor selection. The essential elements of the patient handling program included strengthening the utilization of the lift team, selecting an equipment vendor, and ensuring effective implementation and follow-through. Multiple meetings were held with the patient handling team to develop polices and procedures around the lift team. This included protocols as to when to call the lift team and the specific training for each team member.

In addition to the lift team, the patient handling team investigated various patient transfer equipment vendors to understand what equipment would work best in which situation and with which patients. This was an extensive process that included interviewing each vendor and interviews with several of the vendor's customers to determine equipment satisfaction and utilization compliance. The team narrowed the vendors down to two and eventually one vendor was selected. The selected vendor program uses a three tiered approach; 1) Administrative, 2) Engineering and 3) Behavioral Modification. Elements of the approach include the following:

- Patient handling equipment

- Guarantee incident reduction of $60 \%$

- Weekly to monthly clinical consultations

- $\quad$ Support for 3 years

- Team implementation program of policies, procedures and processes
- Ergonomics training to raise awareness

- Tracking and quantifying the program's financial impact

- Mentoring for equipment utilization

- Best practices designed to specify levels of patient's activity and mobility

A breakdown of the general program expenses (Table 4) show that most of the resources went to equipment followed by training and regular followup by the vendor. Equipment purchased included ceiling lifts, repositioning slings, Maxi-slides, etc. The areas included in the program included all nursing floors and units, all procedural areas (e.g. surgery), rehab hospital, home health, hospice, and out patient centers. The ergonomist assisted periodically with applicable recommendations to strengthen the core elements of the program.

Table 4

Breakdown of patient handling program spend by category.
\begin{tabular}{|ll|}
\hline CATEGORY & COST \\
Equipment and start up supplies & $\$ 754,735$ \\
Training, consulting and follow up & $\$ 187,242$ \\
$\begin{array}{l}\text { Expense for mounting of battery charg- } \\
\text { ers, shelving and laundry carts }\end{array}$ & $\$ 9,383$ \\
$\begin{array}{l}\text { Printing, supplies and other expenses } \\
\text { related to training and kick off fair }\end{array}$ & $\$ 1,774$ \\
\hline
\end{tabular}

Departments began using the equipment in June 2009 and the patient handling injuries were immediate reduced from 38 direct patient handling injuries in the first 6 months of 2009 to only 1 in the month after implementation. The one reported injury in 2009 was the result of an untrained nurse practioner in the emergency center. This area was left out of the initial training and the team quickly brought the training to these employees. In addition, after the initial equipment implementation, the team worked with human resources and nursing leadership to develop an accountability program so that employee use or non-use of the equipment would be measured on their annual performance reviews.

With the assistance of the lift team, implementation of lift equipment, monthly compliance audits to ensure lift equipment utilization and accountability elements, the hospital went from and average of 88 to only 13 patient handling injuries per year, representing an $84 \%$ annualized reduction in patient handling frequency. Of those 13 patient handling injuries that were reported in 2010, seven (7) were flagged as avoidable if the lift equipment would have been utilized. Clearly compliance with the equipment imple- 
mentation remains a challenge in the required scenarios for employees exposed to patient transfers.

\section{$3.35 S$}

$5 \mathrm{~S}$ is a lean program that focuses on eliminating waste and establishing standards for a visual workplace. The rationale for implementing a $5 \mathrm{~S}$ program was that by establishing a facility that is lean and has what it needs, it would control inherent safety and ergonomic hazards. For example, floor level storage was a prevalent issue which created trip/fall and lifting related injuries. In addition, equipment like surgical carts, surgical supplies, beds and IV poles to name few were often stored in hallways creating trip/fall exposures to employees, patients and visitors while increasing the amount of search time to find items since locations rotated without designated storage areas. The warehouse also created issues for the floors due to slow delivery times. This led to excessive (24-30) emergency supply orders that taxed the Supply Chain and thus created a "hording" mentality leading to more lifting and trip/fall exposures. The rush to get into compliance was also a concern in that the hospital needed a strategy to ensure the physical space was in compliances $24 / 7$ to any type of outside inspection it may encounter over the course of a year.

The 5S program, developed by Hiroyuki Hirano, is considered by many to be the first step in Total Quality Management. There are 5 steps or pillars that are executed in sequence which are 1) Sort/Organization, 2) Set in Order, 3) Shine, 4) Standardization and 5) Self-Discipline (Table 5). In the 4Q of 2009 a $5 \mathrm{~S}$ Steering Committee was established and outlined the departments and completion dates for the pilot through a signed $5 \mathrm{~S}$ charter. The program pilot was administered to six initial departments as follows:

1. Surgery/OR Supplies

2. Supply Chain

3. Pharmacy

4. Lab, Facilities

5. Clinical Engineering

6. Patient Care Services.

Employees were trained by the ergonomist during each phase of the program and assisted with the implementation through periodic inspections/audits. Once the group of six completed the phase they were certified to continue to the next phase.

The participating groups improved their overall visual appeal, eliminated hallway and floor level storage, eliminated waste and clutter and created visually appealing work areas with less safety and ergo- nomic hazards while increasing efficiency. In 2011 additional departments will roll out $5 \mathrm{~S}$. This process will continue until the whole enterprise is trained and completes the program.

Table 5

5S Phases, description, completion dates set in the charter and key results achieved.

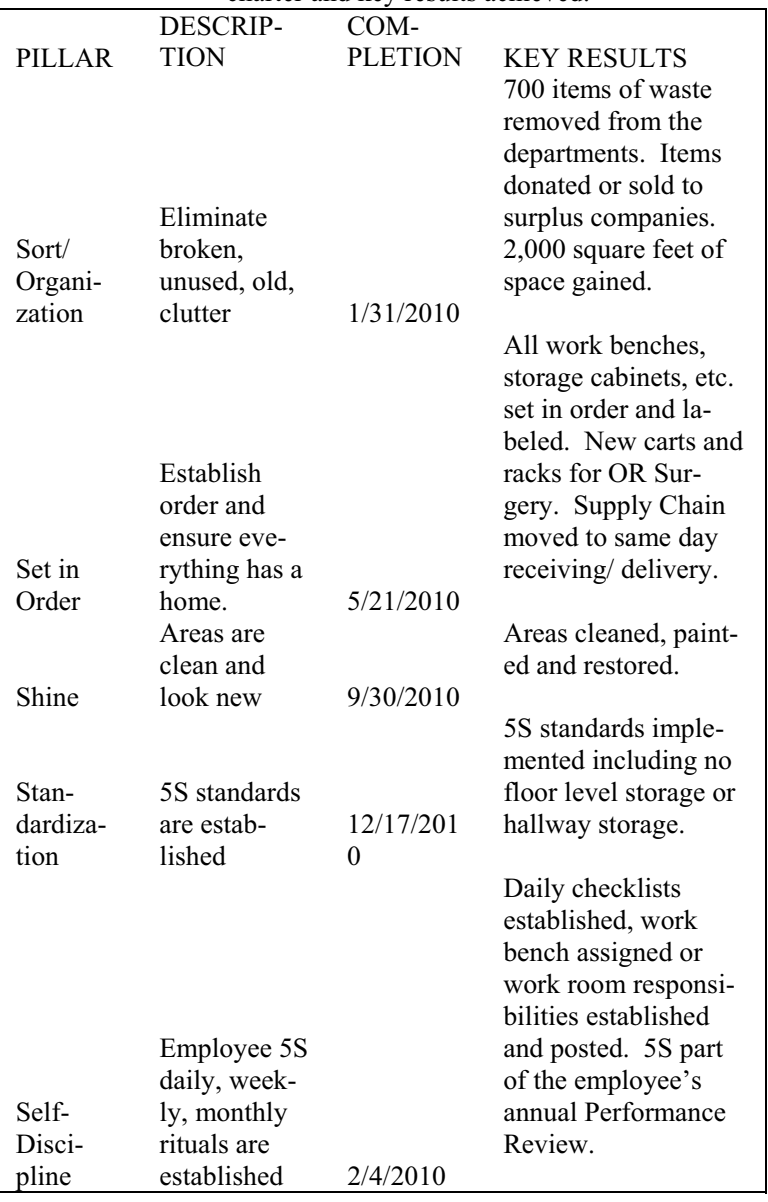

\section{Conclusions}

As a result of the 4 year effort, the hospital has experienced a steady decline in the number of accident reported. In the pre-implementation years the hospital was averaging 43 employee injuries per month. In 2010, the hospital averaged 15 claims per month representing a $65.11 \%$ reduction. While there is still work to accomplish to further reduce the claims, the transformation of this hospital and embracing a proactive, participatory designed programs had produced significant results in eliminating hazards, reducing employee injuries, decreasing waste, and improving the visual appeal of the organization. 